INTERNATIONAL JOURNAL
PHARMACEUTICAL SCIENCES
RESEARCH

Received on 03 September, 2012; received in revised form, 15 October, 2012; accepted, 28 December, 2012

\title{
CHRONOTHERAPY: A REVIEW
}

Manpreet Kaur* and Rajni Bala

Chitkara College of Pharmacy, Chitkara University, Rajpura- 140 401, Punjab, India

Keywords:

Chronotherapy, Pulsatile drug delivery system, Circardian Rhythm,

Chronopharmaceutics, Time Dependent Release

Correspondence to Author:

\section{Manpreet Kaur}

Chitkara College of Pharmacy, Chitkara University, Rajpura- 140 401, Punjab, India

E-mail: manpreet_arora@rocketmail.com

\section{ABSTRACT}

Chronotherapy refers to the use of circadian, ultradian, infradian \& seasonal or other rhythmic cycles in the application of therapy. There are number of conditions which show a circadian pattern and advantage could be taken by timing and adjusting the administration of drugs according to the circadian rhythm of the disease. Some of the conditions, which may be significantly benefited, are hypertension, myocardial infarction, bronchial asthma, peptic ulcer, arthritis, duodenal ulcer, diabetes, neurological disorder, cancer and hypercholesterolemia. Chronotherapy can be classified into time controlled systems wherein the drug release is controlled primarily by the delivery system, stimuli induced PDDS in which release is controlled by the stimuli, such as the $\mathrm{pH}$ or enzymes present in the intestinal tract or enzymes present in the drug delivery system and externally regulated system where release is programmed by external stimuli like magnetism, ultrasound, electrical effect and irradiation.

INTRODUCTION: Circadian rhythm regulates many body functions in humans, like metabolism, physiology, behaviour, sleep patterns, hormone production, etc. The release of some drugs is preferred in pulses i.e. the release of drug as a "pulse" after a lag time has to be designed in such a way that a complete and rapid drug release should follow the lag time.

Lag time is defined as the time between when a dosage form is placed into an aqueous environment and the time at which the active ingredient begins to release from the dosage form. These systems are also called time-controlled as the drug released is independent of the environment ${ }^{1}$.

A single dosage form provides an initial dose of drug followed by one release-free interval, after which second dose of drug is released, which is followed by additional release-free interval and pulse of drug release $^{2}$.
Chronopharmaceutics is a branch of pharmaceutics (science and technology of drug dosage forms) meant to the design and evaluation of drug delivery systems that release a bioactive agent at a rhythm that ideally matches in real time the biological requirement for a given disease therapy or prevention 'Chronopharmaceutic Drug Delivery System' uses the basic concepts of human chronobiology and the rhythm dependence of certain disease states and the pharmacodynamics of medications.

The drug therapy can be optimized by tailoring the dosing schedule based on chronobiological pattern.

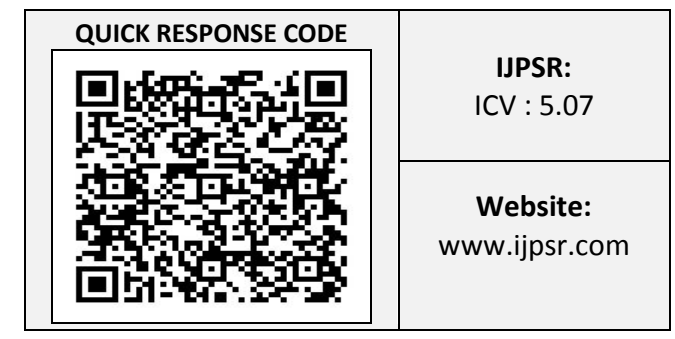


The safety and efficacy of the drug is achieved by coordinating the peak plasma concentration of the drug with circadian rhythm of the body ${ }^{3}$.

Chronotherapeutics is the discipline concerned with the delivery of drugs according to the intrinsic activities of a disease over a certain period of time because the biochemical, physiological and pathological variations over a $24 \mathrm{~h}$ period in humans have occurred ${ }^{4}$.

Different types of the rhythms affecting human body:
Ultradian: these are the cycles shorter than a day. E.g. 90 minutes sleep cycle

Circadian: This lasts for over 24 hours. E.g. sleeping and waking patterns.

Infradian: cycles longer than 24 hours. E.g. monthly menstruation

Seasonal: such as seasonal affective disorder (SAD), this causes depression during the short days of winter in susceptible people ${ }^{5}$.

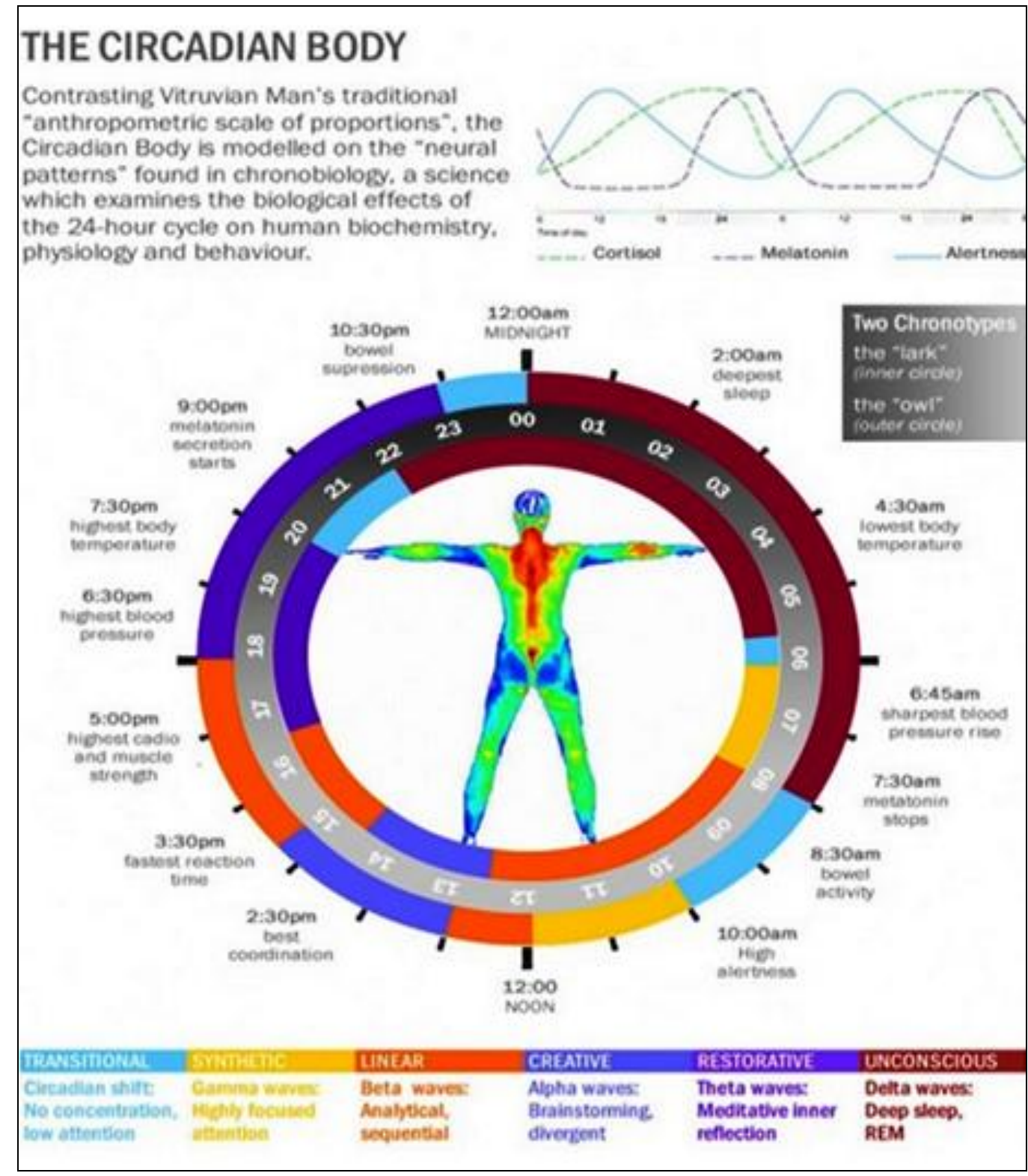

The shift from conventional sustained release approach to modern pulsatile delivery of drugs can be credited to the following reason(s):
1. First pass metabolism: Some drugs, such as beta blockers, and salicylamide, undergo extensive first pass metabolism and require fast drug input to saturate metabolizing enzymes in order to minimize pre-systemic metabolism. 
Thus, a constant/sustained oral method of delivery would result in reduced oral bioavailability.

2. Biological tolerance: Continuous release drug plasma profiles are often accompanied by a decline in the pharmacotherapeutic effect of the drug, e.g., biological tolerance of transdermal nitroglycerin.

3. Special chronopharmacological needs: Circadian rhythms in certain physiological functions are well established. It has been recognized that many symptoms and onset of disease occur during specific time periods of the 24 hour day, e.g., asthma and angina pectoris attacks are most frequently in the morning hours.

4. Local therapeutic need: For the treatment of local disorders such as inflammatory bowel disease, the delivery of compounds to the site of inflammation with no loss due to absorption in the small intestine is highly desirable to achieve the therapeutic effect and to minimize side effects.

5. Gastric irritation or drug instability in gastric fluid: For compounds with gastric irritation or chemical instability in gastric fluid, the use of a sustained release preparation may exacerbate gastric irritation and chemical instability in gastric fluid.

6. Drug absorption differences in various gastrointestinal segments: In general, drug absorption is moderately slow in the stomach, rapid in the small intestine, and sharply declining in the large intestine. Compensation for changing absorption characteristics in the gastrointestinal tract may be important for some drugs. For example, it is rational for a delivery system to pump out the drug much faster when the system reaches the distal segment of the intestine, to avoid the entombment of the drug in the faeces ${ }^{6}$.

\section{Advantages of Chronotherapy:}

- Chronotherapy is drug-free

- It is more effective when a person sleeps for several hours.
- While Chronotherapy patients often fall asleep this improves their condition and confidence as well.

- It is different from other treatments because it got the beginning, middle, and an end. So one can predict easily the point at which it will work.

- It gives a new schedule like getting up and sleeping early which will be quite unusual for some days but it will give a period to adjust psychologically $^{7}$

\section{Disadvantages of Chronotherapy:}

- It develops a non 24 hours sleep wake syndrome after the treatment as the person sleeps for over 24 hours during the treatment. It's not quite common but the degree of risk is not known.

- Person may also be deprived of sleep sometimes.

- Person become less productive during chronotherapy and staying awake till the other schedule might be bit uncomfortable.

- Person will have to take some time off from your busy normal schedule as its time taking therapy.

- Medical supervision is mandatory for this therapy and regular consulting of sleep specialists is recommended.

- Person has to keep himself awake till the next sleep schedule so he have to get himself busy so that he stay awake till the other schedule.

- Person undergoing therapy may feel unusually hot or cold sometimes.

- Patient needs to consult the doctor regularly to avoid side effects ${ }^{7}$ 


\section{Chronotherapy in various diseases:}

1. Asthma: It is characterized by airway inflammation resulting in hyper responsiveness of lower respiratory tract to various environmental stimuli. Airway resistance increases progressively at night in the patient. Asthma is the most common disease in which the large circadian variation occurs with respect to time. There is an increased incidence of asthma during the early-morning hours. The symptoms of asthma occur 50 to 100 times more at night. The exacerbation of asthma during the night represents the changing status of biological functioning due to circadian rhythms in bronchial patency; airways hyper reactivity to acetylcholine, histamine, and house dust; and plasma cortisol, epinephrine, histamine, and cyclic AMP. Once daily dosing of inhaled glucocorticosteroid ciclesonide, sustained release theophylline, transdermal tulobuterol patch found to be effective in case of nocturnal asthma ${ }^{8}$.

2. Cardiovascular disease: In cardiovascular disease capillary resistance and vascular reactivity are higher in the morning and decreases later in the day. Increased platelet aggregation and decreased fibrinolytic activity in the morning leads to relative hypercoagulability of the blood. Also BP is at its lowest during the sleep cycle and rises steeply during the early morning awakening period. These observations shows that myocardial ischemia, angina pectoris, acute myocardial infarction, congestive cardiac failure and sudden cardiac death are also unevenly distributed during the 24 $h$ with greater expected events during the initial hours of the daily activity span, in the late afternoon or early evening.

Both sympathetic activity and the Reninangiotensin-aldosterone axis peak in the early morning hours. External factors affecting ANS including physical activity, emotional state, meal and sleep/wake routine also contribute to variations. Currently, there are chronotherapeutic antihypertensive products like oral nitrates, calcium channel blocker and $\beta$-adrenoceptor antagonist whose both pharmacokinetics and pharmacodynamics get influenced by circadian rhythm are available with novel drug delivery systems, releasing drug during the vulnerable period of 6 am to noon upon administration of medications at $10 \mathrm{pm} .9,10,11$.

3. Cancer: Human and animal studies suggest that chemotherapy may be more effective and less toxic if cancer drugs are administered carefully at selected times that take advantage of tumour cell cycles while less toxic to normal tissue. The rhythmic circadian changes in tumour blood flow and cancer growth are relevant both when tumours are small and growing most rapidly and when they are larger and growing more slowly. Circadian chemotherapy timing meaningfully affects drug toxicity patterns and severity, maximum tolerated dose, average dose intensity, tumour response quality and frequency and the survival of patients with cancer.

The pharmacologic and pharmacokinetic properties of the drug, rhythmic changes in DNA and RNA synthesis, RNA translational activity and mitotic activity may influence tumour cell susceptibility. The cancer chronogenetic therapy found to be effective in tumour suppression in vivo. For example, it has been shown that CLOCK genes dictate sensitivity to the anticancer drug cyclophosphamide. ${ }^{12,13}$.

4. Peptic ulcer: Many of the functions of the gastrointestinal tract are subject to circadian rhythms: gastric acid secretion is highest at night. While gastric, small bowel motility and gastric emptying are all slower at night. Suppression of nocturnal acid is an important factor in duodenal ulcer healing. Therefore, for active duodenal ulcer, once daily at bedtime is the recommended dosage regimen for $\mathrm{H} 2$ antagonists. Bedtime $\mathrm{H} 2$-receptor blockade using Chronotherapy overcome problems of sustained or profound decrease of 24-h intragastric acidity including the threat of enteric infection and infestation, potential bacterial overgrowth with possible $\mathrm{N}$-nitrosamine formation 14 .

5. Arthritis: Rheumatoid arthritis can be distinguished from osteoarthritis by the time of day when the patient's joints are most painful and morning stiffness is characteristic feature of 
rheumatoid arthritis whereas symptoms are often worse in the afternoon and worse in evening in osteoarthritis. Non-steroidal anti-inflammatory drugs are taken for relieving the morning pain and stiffness of rheumatoid arthritis so the medicines are taken late at night and it is better for the treatment. The new cyclooxygenase-2 inhibitors are effectively relieve the osteoarthritis symptoms when taken in the morning and better results are obtained in rheumatoid arthritis when small part of the dose is taken in the evening.

Chronotherapy for all forms of arthritis uses standard treatment that includes the non-steroidal anti-inflammatory drugs and corticosteroids but in the treatment the dosages time are match with the rhythms of disease which are timed to ensuring that the highest blood levels of the drug coincide with peak pain. ${ }^{15,16}$.

6. Hypercholesterolemia: The higher rates of cholesterol intake during the hypercholesterolemia and hepatic cholesterol genesis occur during the evening hours even in the fasting state also. The free cholesterol levels have been reported to be lowest at 2 p.m. to 6 p.m. and peak at $6 \mathrm{am}$ and morning versus evening administration of HMG-CoA reductase antagonists. 17.

7. Allergic Rhinitis: Symptoms of allergic rhinitis (e.g. nasal congestion, sneezing, running nose) are typically more severe in the early-morning hours. If the administration of the drug can be matched with the biological time structure which have the peak with the biological time structure which have the peak pharmacologic activity are matching the time of greatest discomfort, optimum relief may be provided at the time when it is needed most of the patient ${ }^{18}$.

8. Mood Disorders: The deprivation of sleep in the half of the night and the timed exposure to day light-intensity and artificial light still experimental therapies, may ease the depression pre menstrual or during menopause and benefit both women and men with seasonal and other mood disorders. Such a variation was not detected in the mood disorders when sustained release dosage forms of nifedipine and isosorbide mononitrate were used. 19

9. Diabetes: In type I diabetes the circadian rhythms of insulin and its action are of physiological interest and clinical importance. So, insulin is released in pulsatile fashion but sometimes it is irregular. Insulin can show its cyclic rhythmicity of 8-30 min which can show the optimal action. The modulators of insulin release and action are secreted in a circadian pattern and impress the mode of insulin release. So difference between maximum and minimum plasma insulin concentration has short-term rhythmicity and complex secondary circadian rhythm is variable early-morning and late-afternoon insulin resistance. ${ }^{20}$.

10. Sleep Disorders: Many biological signals like sleep disorder occurring in the central and autonomous nervous systems this shows the complex time structure with rhythmic and pulsatile variations in multiple frequencies. Sleep mainly consists of a rhythmic combination or circadian changes in physiological, biochemical and psychological processes. When the circadian rhythm is disturbed or when the individual physiological or psychological processes are abnormal during sleep it may result in a variety of disorders. The circadian rhythm disturbances also differ from person to person and identification of the individual variation would be important in dealing with certain sleep disorders ${ }^{21}$.

11. Epilepsy: The circadian rhythm also plays a significant role in seizures of epilepsy. The influence of the biological clock on seizure of some partial seizures has been found in animals or humans. The behavioral chronobiology provides the detection of new regulation processes that concerns central mechanisms of epilepsy because the circadian psycho physiological patterns of epilepsy show dynamic biological systems which show some intermodulating endogenous processes between observation and seizure susceptibility. Such chronobiologic studies applied to epileptic behaviour and this suggests the development of new heuristic aspects in the field of comparative psychophysiology 22 . 
12. Alzeimer's Disease: The change of circadian rhythm is also seen in patients with Alzheimer's disease. Individuals with Alzheimer's symptoms show less diurnal motor activity and higher percentage of nocturnal activity which show the lower inter daily stability of motor activity and activity of macrophages peak time than normal healthy individuals. The core body temperature is also higher in patients and the circadian abnormalities are seen together with cognitive and functional deterioration in this disease ${ }^{23}$.
13. Parkinson's Disease: Parkinson's disease discloses many alterations in circadian rhythm of blood pressure; amplified diurnal blood pressure variability and postprandial hypotension are due to autonomic dysfunction. But existence of circadian rhythm in this disease has not been evaluated in clinical data because the daily fluctuations of motor activity pattern of the phase of the disease and the subsequent role of drugs are difficult to estimate ${ }^{24}$.

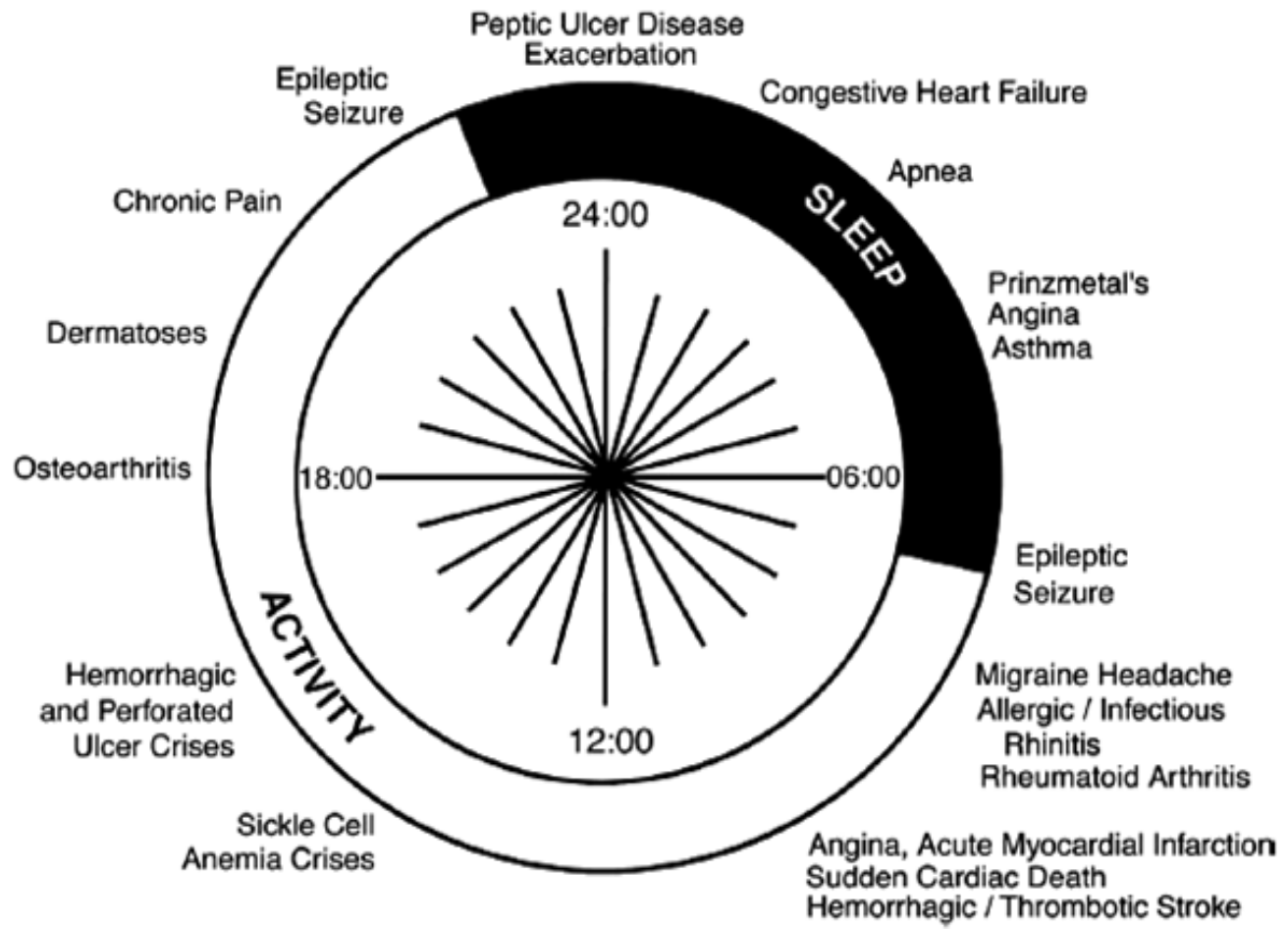

DISPLAY IN THE FORM OF A 24H CLOCK DIAGRAM OF THE APPROXIMATE TIME, IN HUMAN FOLLOWING THE DIURNAL ACTIVITY/NOCTURNAL SLEEP ROUTINE, WHEN SYMPTOMS OR EVENTS OF DISEASES ARE WORST OR MOST FREQUENT

\section{Diseases that require Pulsatile Delivery:}

\begin{tabular}{ccc}
\hline Chronological behaviour & Drugs used & Diseases \\
\hline Acid secretion is high in afternoon and at night & H2 Blockers & Peptic Ulcer \\
\hline Precipitation of attacks during night or at early morning & B2 Antagonist, Antihistaminics \\
\hline $\begin{array}{c}\text { BP is at its lowest during the sleep cycle \& rises steeply during } \\
\text { the early morning }\end{array}$ & $\begin{array}{c}\text { Nitroglycerine, calcium channel } \\
\text { blockers, ACE Inhibitors }\end{array}$ \\
\hline Pain in the morning \& more pain in the night & NSAIDS, Glucocorticoids & Sardiovascular disease \\
\hline Increase the blood sugar level after meal & Arthritis \\
\hline $\begin{array}{c}\text { Cholesterol synthesis is generally high during night time than } \\
\text { day time }\end{array}$ & HMG CoA reductase inhibitors & Hypercholesterolaemia \\
\hline
\end{tabular}

\section{Ideal characteristics of Chronotherapy:}

- Non-toxic within approved limits of use
- Should have a real-time and specific triggering biomarker for a given disease state

- Should have a feed- back control system (e.g. self-regulated an adaptative capability to 
circadian rhythm and individual patient to differentiate between awake - sleep status)

- Biocompatible and biodegradable, especially for parenteral administration

\section{MARKETED TECHNOLOGIES}

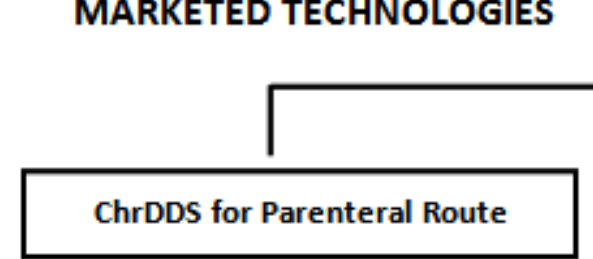

Infusion Pump

Microfabrication technology

Triggable Liposome

Diffucaps ${ }^{x}$
- Easy to manufacture at economic cost,

- Easy to administer in to patients in order to enhance compliance to dosage regimen ${ }^{25}$

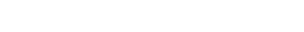

\section{ChrDDS for Oral Route}

Chronotropic ${ }^{\mathrm{TM}}$ Technology

Egalet $^{*}$

OROS $^{x}$ or Chronoset ${ }^{\mathrm{TM}}$

Contin $^{\mathrm{TM}}$

Codas $^{x}$

Ceform ${ }^{x}$

GeoClock $^{\mathrm{TM}}$

Port $^{\mathrm{TM}}$ System

Three Dimensional Printing ${ }^{x}$

TIMERx ${ }^{*}$ Technology

\begin{tabular}{|c|c|c|c|c|}
\hline Technology & Mechanism & Proprietary name \& Dosage form & API & Disease \\
\hline OROS* & Osmotic mechanism & Covera-H5*: XL tablet & Verapamil HCl & Hypertension \\
\hline $\begin{array}{c}\text { Three Dimensional } \\
\text { printing* }\end{array}$ & $\begin{array}{c}\text { Externally regulated } \\
\text { system }\end{array}$ & Their Form* & Diclofenac sodium & Inflammation \\
\hline DIFFUCAPS* & Multiparticulate system & Innopran*: XL tablet & $\begin{array}{l}\text { Verapamil HCl, } \\
\text { Propanolol HCl }\end{array}$ & Hypertension \\
\hline Pulsincap TM & Rupturable system & Pulsincap TM & Dofetilide & Hypertension \\
\hline
\end{tabular}

\section{Classification of Chronotherapeutic Drug Delivery} Systems: Pulsatile drug delivery system can be broadly classified into three classes;

\section{Time controlled drug delivery}

\section{Stimuli induced drug delivery}

3. Externally regulated drug delivery

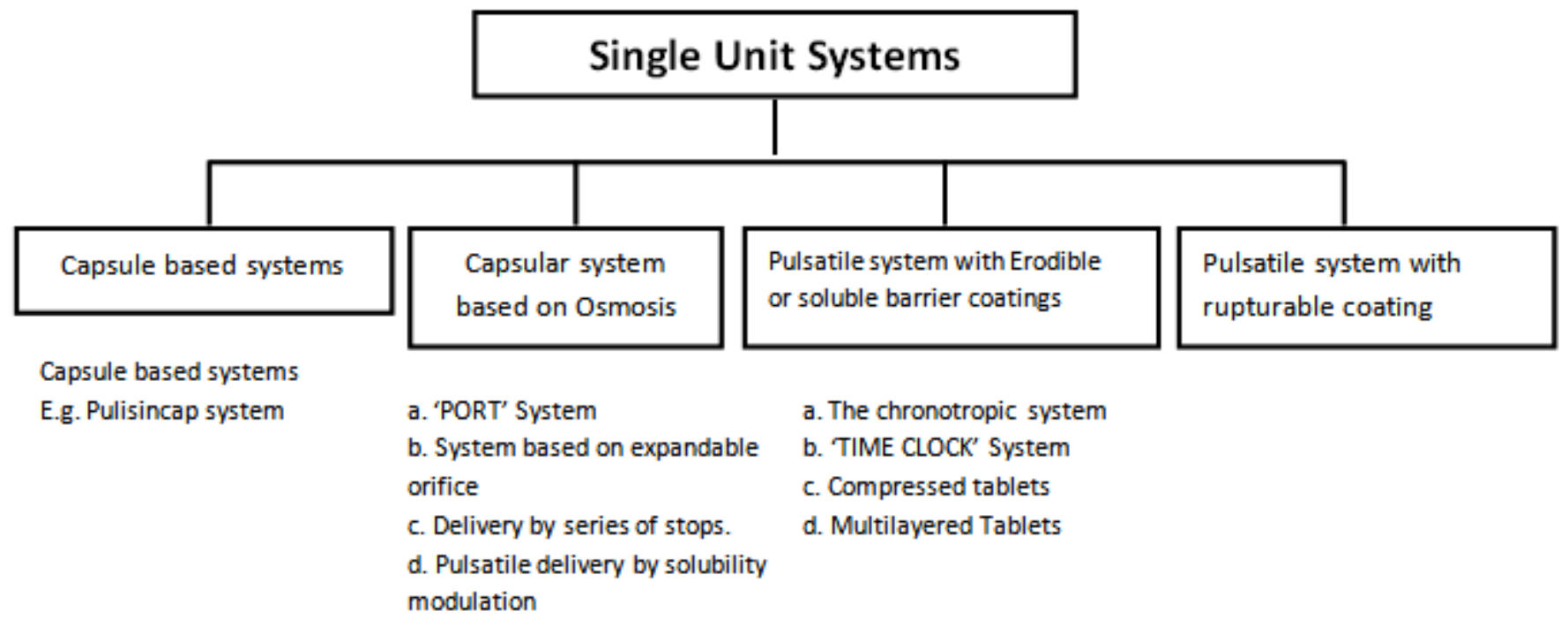




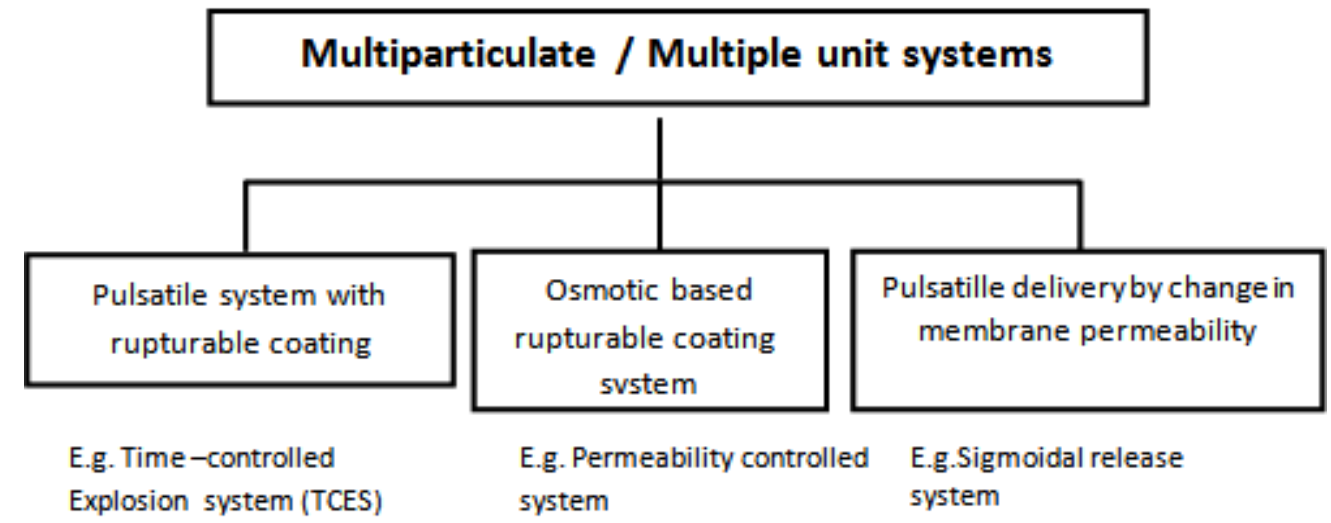

\section{Single Unit Pulsatile Systems:}

\section{ENTERIC COATED PULSINCAP}

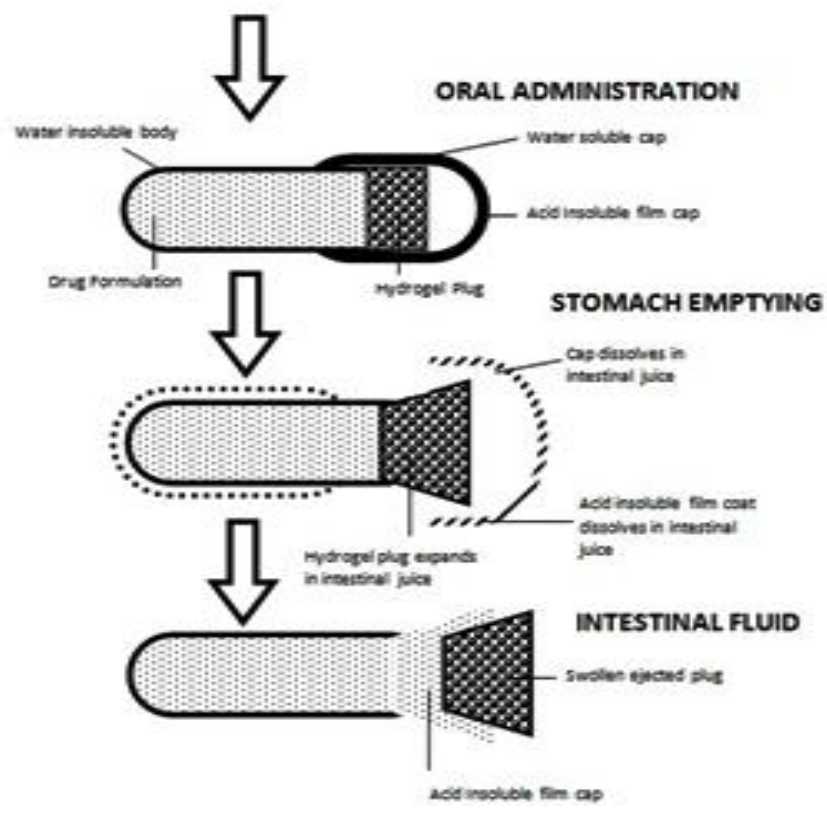

1. Capsule based systems: Single-unit systems are mostly developed in capsule form. The lag time is controlled by a plug, which gets pushed away by swelling or erosion, and the drug is released as a "Pulse" from the insoluble capsule body. The lag time is controlled by manipulating the dimension and the position of the plug. Polymers used for designing of the hydrogel plug:
A. Insoluble but permeable and swellable polymers (e.g., polymethacrylates)

B. Erodible compressed polymers (e.g., hydroxy propylmethyl cellulose, polyvinyl alcohol, Polyethylene oxide)

C. Congealed melted polymers (e.g., saturated polyglycolated glycerides, glyceryl monooleate)

D. Enzymatically controlled erodible polymer (e.g., pectin) 26, 27

\section{Capsular system based on Osmosis:}

1) 'PORT' System: The Port system consists of a capsule coated with a semi permeable membrane. Inside of the capsule contains an insoluble plug consisting of osmotically active agent and the drug formulation. When the capsule comes in contact with the dissolution fluid, the semi permeable membrane allows the entry of water, which causes the pressure to develop and the insoluble plug expels after a lag time. E.g. Methylphenidate is used in the treatment of attention deficit hyperactivity disorder as the pulsatile port system. This system avoided second time dosing, which was beneficial for school children during daytime. 28,29

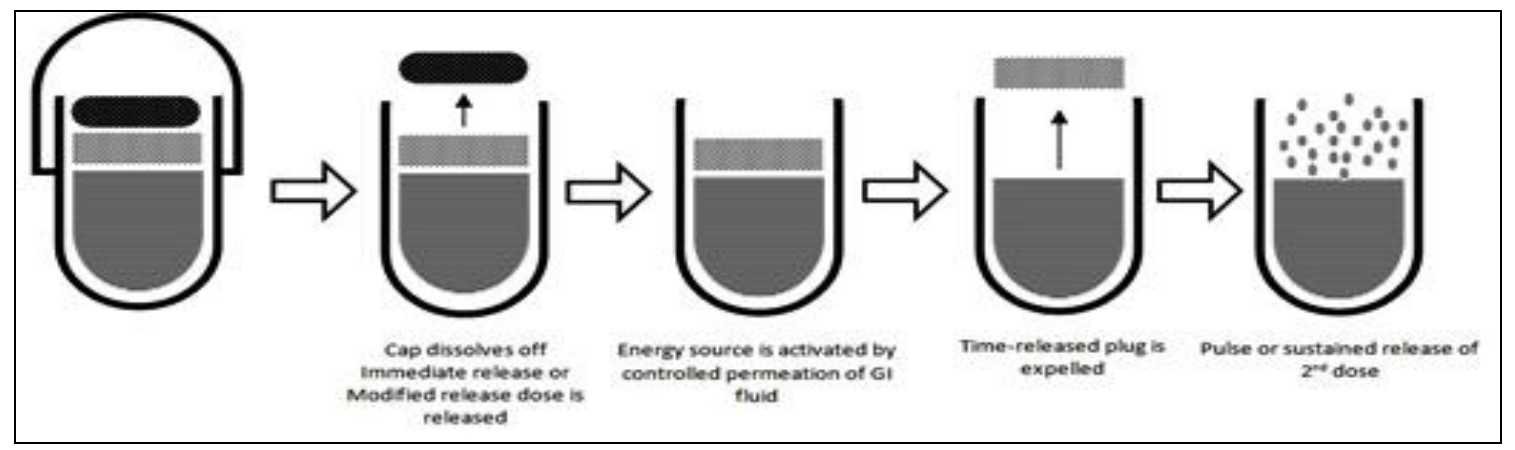

DRUG RELEASE MECHANISM FROM PORT SYSTEM 
2) System based on Expandable Orifice: To deliver the drug in liquid form, an osmotically driven capsular system is developed in such a way that the liquid drug is absorbed into highly porous particles, which releases the drug through an orifice of a semi permeable capsule supported by an expanding osmotic layer after the barrier layer is dissolved.

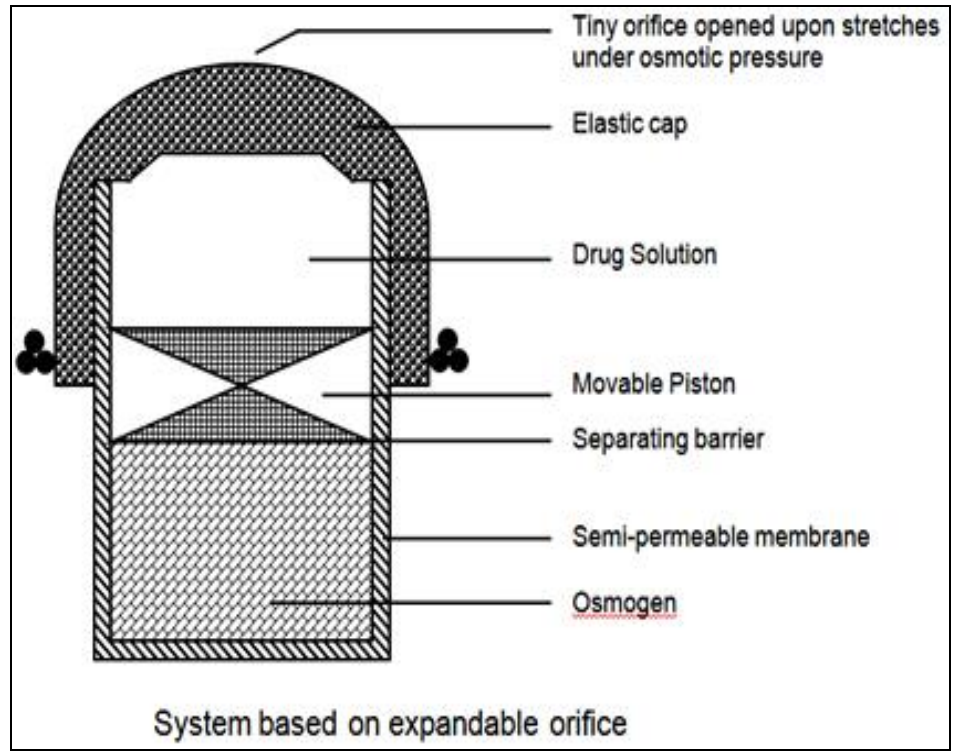

The orifice is small enough so that when the elastic wall relaxes, the flow of the drug through the orifice stops, but when the elastic wall is distended beyond threshold value, the orifice expands sufficiently to allow drug release at a required rate. E.g. Elastomers, such as styrene-butadiene copolymer have been suggested ${ }^{30,31}$.

3) Delivery by series of stops: This system is used for implantable capsules. The capsule contains a drug and a water absorptive osmotic engine that are placed in compartments separated by a movable partition. The pulsatile delivery is achieved by a series of stops along the inner wall of the capsule. These stops obstruct the movement of the partition but are overcome in succession as the osmotic pressure rises above a threshold level ${ }^{32}$.

4) Pulsatile delivery by solubility modulation: These systems contain a solubility modulator for pulsed delivery of variety of drugs. The system is especially developed for delivery of salbutamol sulphate.
The compositions contain the drug (salbutamol sulphate) and a modulating agent (sodium chloride). The amount of $\mathrm{NaCl}$ is less than the amount needed to maintain saturation in a fluid that enters the osmotic device. The pulsed delivery is based on drug solubility. Salbutamol has solubility of $275 \mathrm{mg} / \mathrm{ml}$ in water and 16 $\mathrm{mg} / \mathrm{ml}$ in saturated solution of $\mathrm{NaCl}$, while $\mathrm{NaCl}$ has solubility of $321 \mathrm{mg} / \mathrm{ml}$ in water, and its saturation solubility is $320 \mathrm{mg} / \mathrm{ml}^{33,34}$.

3. Pulsatile system with Erodble or soluble barrier coatings: Most of the pulsatile drug delivery systems are reservoir devices coated with a barrier layer. This barrier erodes or dissolves after a specific lag period, and the drug is subsequently released rapidly from reservoir core. The lag time depends on the thickness of the coating layer. ${ }^{35}$.

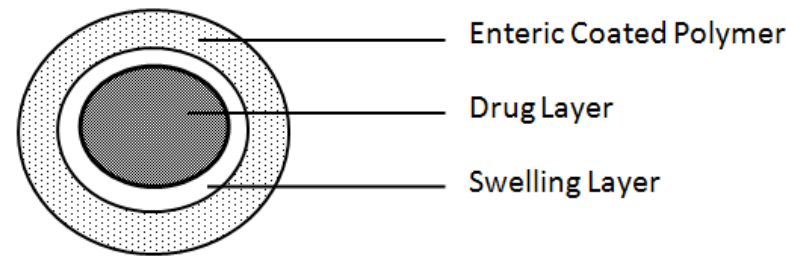

1) The chronotropic system: The Chronotropic ${ }^{\circledR}$ system consists of a drug-containing core coated by hydrophilic swellable hydroxypropyl methyl cellulose (HPMC), which is responsible for a lag phase in the onset of release. In addition, through the application of an outer gastric-resistant enteric film, the variability in gastric emptying time can be overcome, and a colon-specific release can be obtained, relying on the relative reproducibility of small intestinal transit time. The lag time is controlled by the thickness and the viscosity grades of HPMC. Both in-vitro and in-vivo lag times correlate well with the applied amount of the hydrophilic retardin polymer. The system is suitable for both tablets and capsules ${ }^{36}$.

2) 'TIME CLOCK' System: The lag time is controlled by varying the thickness of the film. After the lag time, i.e., the time required for rehydration, the core immediately releases the drug. This system has shown reproducible results in vitro and in vivo. 
The effect of low calorie and high calorie meal on the lag time was studied using gamma scintigraphy. The mean lag time of drug release was 345 and 333 min respectively. ${ }^{37,} 38$.

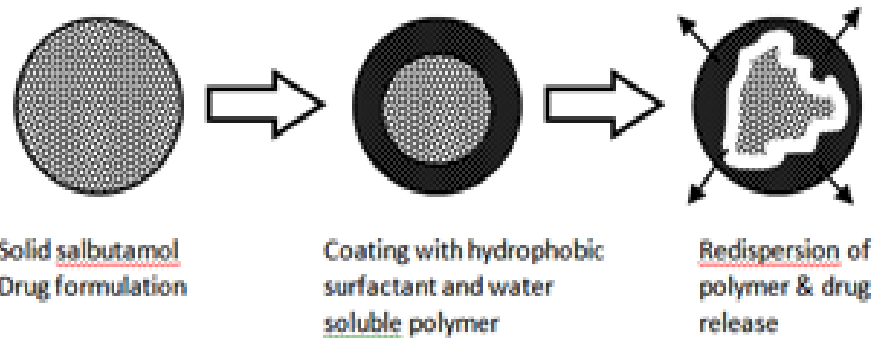

3) Compressed Tablets: Compression coating involves direct compression of both the core and the coat, obviating needs for separate coating process and use of coating solutions. The outer tablet of the compression-coated tablet provides the initial dose, rapidly disintegrating in the stomach and the inner layer is formulated with components that are insoluble in gastric media but are released in the intestinal environment. Materials such as hydrophilic cellulose derivates can be used. The major drawbacks of the technique are that relatively large amounts of coating materials are needed and it is difficult to position the cores correctly ${ }^{39}$.

Press-coated pulsatile drug delivery systems:

- It is used to protect hygroscopic, light-sensitive, oxygen labile or acid-labile drugs.

- These are relatively simple and cheap.

- These can involve direct compression of both the core and the coat.

- Materials such as hydrophobic, hydrophilic can be used in press-coated pulsatile drug delivery system.

- It involves compression which is easy on laboratory scale.

- These formulations release drug after "lagtime".
- These formulations can be used to separate incompatible drugs from each other or to achieve sustained release. ${ }^{40}$.

4) Multilayered Tablets: A release pattern with two pulses is obtained from a three layered tablet containing two drug containing layers separated by a drug-free gellable polymeric barrier layer. ${ }^{41}$.

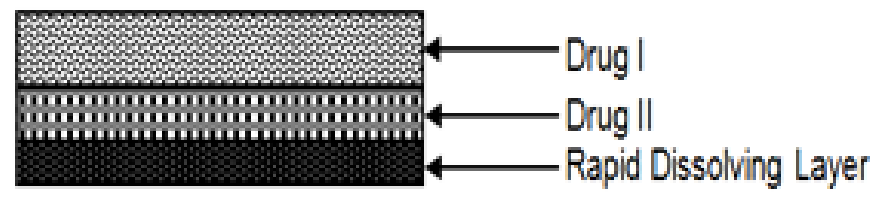

4. Pulsatile system with rupturable coating: These systems depend on the disintegration of the coating for the release of drug. The pressure necessary for the rupture of the coating can be achieved by the effervescent excipients, swelling agents, or osmotic pressure. An effervescent mixture of citric acid and sodium bicarbonate was incorporated in a tablet core coated with ethyl cellulose. The carbon dioxide developed after penetration of water into the core resulted in a pulsatile release of drug after rupture of the coating. The release may depend on the mechanical properties of the coating layer ${ }^{42}$.

1) Pulsatile system based on Rupturable Coating: E.g. Time-controlled Explosion system (TCES): This is a multiparticulate system in which drug is coated on non-pareil sugar seeds followed by a swellable layer and an insoluble top layer. The swelling agents used include Superdisintegrants like sodium carboxymethyl cellulose, sodium starch glycollate, L-hydroxypropyl cellulose. Polymers like polyvinyl acetate, polyacrylic acid, polyethylene glycol, etc ${ }^{43}$.

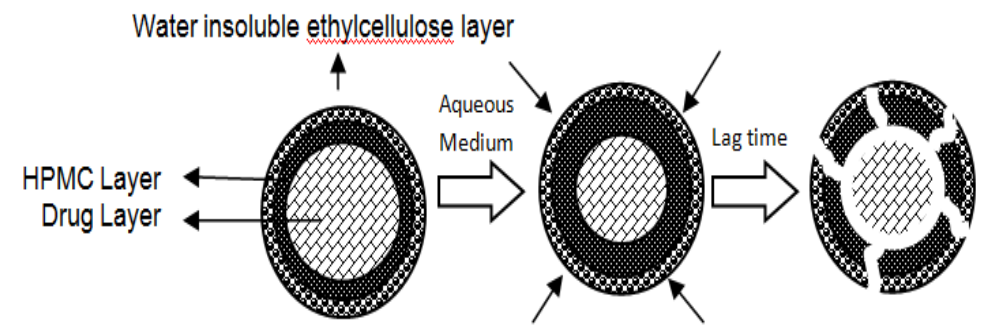


2) Osmotic based rupturable coating system: This system is based on a combination of osmotic and swelling effects. The core containing the drug, a low bulk density solid and/or liquid lipid material (e.g., mineral oil) and a disintegrant was prepared. This core was then coated with cellulose acetate. Upon immersion in aqueous medium, water penetrates the core displacing lipid material. After the depletion of lipid material, internal pressure increases until a critical stress is reached, which results in rupture of coating ${ }^{44}$.

3) Pulsatile delivery by change in membrane permeability: The permeability and water uptake of acrylic polymers with quaternary ammonium groups can be influenced by the presence of different counter-ions in the medium. 48 Several delivery systems based on this ion exchange have been developed. Eudragit RS 30D is reported to be a polymer of choice for this purpose. It typically contains positively polarized quaternary ammonium group in the polymer side chain, which is always accompanied by negative hydrochloride counter-ions.

The ammonium group being hydrophilic facilitates the interaction of polymer with water, thereby changing its permeability and allowing water to permeate the active core in a controlled manner. This property is essential to achieve a precisely defined lag time ${ }^{45,46}$.

\section{Stimuli-induced Pulsatile Release:}

1. Temperature-induced pulsatile release: Thermoresponsive hydrogels have been investigated as possible drug delivery carriers for stimuli responsive drug delivery systems. Thermoresponsive polymeric micelle systems, the properties and biological interests of polymeric micelles make them a most noteworthy candidate as drug carrier for the treatment of cancer. The polymeric micelle is composed of amphiphilic block copolymers exhibiting a hydrophobic core with a hydrophilic corona. ${ }^{47,48}$.

\section{Chemical stimuli-induced pulsatile release:}

1) Glucose-responsive Insulin Release Devices: A decrease in or the absence of insulin secretion from pancreatic islets is the cause of diabetes mellitus. Diabetes mellitus patients suffer long term from a gradual decline in the efficiency of various organs, such as the occasional loss of eyesight. Several systems have already been developed which are able to respond to glucose concentration changes. Glucose oxidase (GOD) catalyzes glucose oxidation. Utilizing this reaction, preparation of two types of gel membrane systems to regulate insulin permeability is done ${ }^{49,50}$.

2) Inflammation-induced pulsatile release: When human beings receive physical or chemical stress, such as injury, broken bones, etc., inflammation reactions take place at the injured sites. At the inflammatory sites, inflammationresponsive phagocytic cells, such as macrophages and polymorph nuclear cells, play a role in the healing process of the injury. During inflammation, hydroxyl radicals $(\mathrm{OH})$ are produced from these inflammation-responsive cells. Yui and co-workers used hyaluronic acid (HA), a linear mucopolysaccharide composed of repeating disaccharide subunits of $\mathrm{N}$-acetyl-Dglucosamine and D-guluronic acid. In the body, $H A$ is mainly degraded either by a specific enzyme, hyaluronidase, or hydroxyl radicals.

Degradation of HA via the hyaluronidase is very low in a normal state of health. Degradation via hydroxyl radicals however, is usually dominant and rapid when $\mathrm{HA}$ is injected at inflammatory sites. Thus, Yui and co-workers prepared crosslinked HA with ethyleneglycol diglycidylether or polyglycerol polyglycidylether. These HA gels degraded only when the hydroxyl radicals were generated through the Fenton reaction between $\mathrm{Fe}^{+2}$ ions and hydrogen peroxide in vitro.

Thus, a surface erosion type of degradation was achieved. When microspheres were incorporated in the HA hydrogels as a model drug, these microspheres were released only 
when hydroxyl radicals induced HA gel degradation. The microsphere release was regulated by the surface erosion type of degradation $51,52,53$.

3) Drug release from Intelligent Gels responding to Antibody concentration: There are numerous kinds of bioactive compounds which exist in the body. Recently, novel gels were developed which responded to the change in concentration of bioactive compounds to alter their swelling/deswelling characteristics. Miyata and co-workers focused on the introduction of stimuli-responsive cross-linking structures into hydrogels. Special attention was given to antigen antibody complex formation as the cross-linking units in the gel, because specific antigen recognition of an antibody can provide the basis for a new device fabrication ${ }^{54,55}$.

\section{4) Electric stimuli-responsive Pulsatile Release:} The combination of developments in several technologies, such as microelectronics and micromachining, as well as the potential need for chronotherapy, have currently assisted the development of electronically assisted drug delivery technologies. These technologies include iontophoresis, infusion pumps, and sonophoresis. Several approaches have also been presented in the literature describing the preparation of electric stimuli-responsive drug delivery systems using hydrogels ${ }^{56}$.

Kishi et al., developed an electric stimuli induced drug release system using the electrically stimulated swelling /deswelling characteristics of polyelectrolyte hydrogels. They utilized a chemomechanical system, which contained a drug model within the polyelectrolyte gel structure. These gels exhibited reversible swelling/shrinking behaviour in response to on-off switching of an electric stimulus. Thus, drug molecules within the polyelectrolyte gels might be squeezed out from the electric stimuli-induced gel contraction along with the solvent flow. To realize this mechanism, poly (sodium acrylate) microparticulate gels containing pilocarpine as a model drug were prepared ${ }^{57}$.

\section{REFERENCES:}

1. Pednekar PP, Parmar CK: Chronopharmaceutical Drug Delivery Systems: An Overview, International Journal Of Current Pharmaceutical Research, Vol 3, Issue 4, 2011

2. Jha N, Bapat S: Chronobiology and chronotherapeutics. Kathmandu University Med. Jour. 2004; 2(8): 384-388.

3. Patel G: Specialized chronotherapeutic drug delivery systems, Pharmainfo.net

4. Sarasija S, Hota A, Colon-specific drug delivery systems, Ind. J. Pharm. Sci., 62(1), 2002, 1-8.

5. Sajan J, Cinu TA, Chacko AJ, Litty J and Jaseeda T: Chronotherapeutics and Chronotherapeutic Drug Delivery Systems, Tropical Journal of Pharmaceutical Research, October 2009; 8 (5): 467-475

6. Saigal N, Baboota S, Ahuja A and Ali J : Site Specific Chronotherapeutic Drug Delivery Systems: A Patent Review, Recent Patents on Drug Delivery \& Formulation 2009, 3, 64-70

7. Srinivas $P$, Sandeep G, Pridhvi AR And Soujanya $Y$ : Chronotherapy- Clock Of Curing, International Journal of Pharma and Bio Sciences, Vol 2/ Issue 3/ Jul-Sept 2011

8. Martin RJ, Banks-Schlegel S. Chronobiology of asthma, Am J Respir Crit Care Med. 1998; 158: 1002- 1007.

9. Muller JE, Tofler GH, Stone PH. Circadian variation and triggers of onset of acute cardiovascular disease. Circulation 1989; 79:733-743

10. Lemmer B. Cardiovascular chronobiology and chronopharmacology. Biological Rhythms in Clinical and Laboratory Medicine 1992; 418- 427.

11. Tofler GH, Brezinski D, Schafer Al, Czeisler CA, Rutherford JD, Willich SN, Gleason RE, Williams GH, Muller JE. Concurrent morning increase in platelet aggregability and the risk of myocardial infarction and sudden cardiac death. J Med 1987; 316: 1514-1518.

12. Hori K, Zhang QH, Li HC, Saito S, Sato Y. Timing of cancer chemotherapy based on circadian variations in tumor tissue blood flow. Int J Cancer 1996; 65:360-364.

13. Levi V. Circadian chronotherapy for human cancers. Lancet Oncol 2001; 2: 307-315.

14. Humphries TJ, Root JK, Hufnagel K. Successful drugspecific chronotherapy with the $\mathrm{H} 2$ blocker famotidine in the symptomatic relief of gastro-esophageal reflux disease. Ann New York Acad Sci 1991; 517-518.

15. Herold M, Gunther R. Circadian rhythm of C-reactive protein in patients with rheumatoid arthritis. Prog Clin Biol Res 1987; 271- 279.

16. Arvidson NG, Gudbjornsson B, Elfman L, Ryden AC, Totterman $\mathrm{TH}$, Hallgren R. Circadian rhythm of serum interleukin- 6 in rheumatoid arthritis. Ann Rheum Dis1994; 53: 521-524.

17. Richard MD, Havel J. Simvastatin: a one-a-day treatment for hypercholesterolemia An Introduction. Am J Med 1989; 87 (Suppl 4): 1S- 59S.

18. Conte U, Giunchedi P, Maggi L, Sangalli ME, Gazzaniga A, Colombo P, Manna A, Ibuprofen delayed release dosage forms: a proposal for the preparation of an in vitro/in vivo pulsatile system, Eur. J. Pharm., 1992; 38(6): 209-212

19. Janugade BU, Patil SS, Patil SV, Lade P D: Pulsatile drug delivery system for chronopharmacological disorders: an overview Review Article Journal of Pharmacy Research 2009;2;1:136

20. Cincotta $\mathrm{AH}$, Meier AH. Circadian rhythms of lipogenic and hypoglycaemic responses to insulin in the golden hamster (Mesocricetus auratus). J Endocrinol 1984; 103: 141-146.

21. Singh R., Sharma P.K. and Malviya R.: Review on Chronotherapeutics - A New Remedy in the Treatment of 
Various Diseases, European Journal of Biological Sciences 2 (3): 67-76, 2010

22. Latha K, Uhumwangho, MU Sunil, SA Srikanth, MV Ramana, Murthy KV : Chronobiology and Chronotherapy of Hypertension - A Review, International Journal of Health Research, September 2010; 3(3): 121-131

23. Auvil-Novak SE. The chronobiology, chronopharmacology, and chronotherapeutics of pain. Annu Rev Nurs Res. 1999; 17: 133153.

24. Mandal AS, Biswas N, Karim KM, Guha A, Chatterjee S, Behera $M$, Kuotsu K.: Drug delivery system based on chronobiology. A review Journal of Controlled Release. 2010;10.

25. Rathod S. : Colon Targeted Pulsatile Drug Delivery : A Review. Pharmainfo net 2007; 5(2), 1-11

26. Bodmeier R. Pulsatile drug release from an insoluble capsule body controlled by an erodible plug. Pharm Res 1998; 15(3): 474-481.

27. Dinaravand RD, Emanele A. Use of thermoresponsive hydrogels for on-off release of molecules. J Control Rel 1995; 221-227.

28. Crison JR, Siersma PR, Taylor MD, Amidon GL. Programmable oral release technology, Port Systems \& Mac226: a novel dosage form for time and site specific oral drug delivery. Proceed Intern Symp Control Rel Bioact Mater 1995; 22: 278279.

29. Rajput $M$, Sharma R, Kumar S, Jamil F, Sissodia N and Sharma S : Pulsatile Drug Delivery System: A Review, International Journal of Research in Pharmaceutical and Biomedical Sciences ISSN: 2229-3701

30. Linkwitz A, Magruder JA, Merrill S. Osmotically Driven Delivery Device with Expandable Orifice for Pulsatile Delivery Effect. US Patent No. 5,318,558; 1994.

31. Survase S, Kumar N: Pulsatile Drug Delivery: Current Scenario. Current Research \& Information on Pharmaceutical Sciences. 2007; 8(2): 27-33.

32. Balaban SM, Pike JB, Smith JP, Baile CA. Osmotically Driven Delivery Devices with Pulsatile Effect. US Patent No. 5209746; 1993.

33. Suthar M, Dr. Patel U, Brahmbhatt $T$, Patel $H$, Bhatt $S$, Kadikar $H$ : Pulsatile Drug Delivery: A Review, Volume 1(1)

34. Patel VP, Desai TR, Matholiya CR, Chhayani RB: Pulsatile Drug Delivery System: A Review, Pharmatutor-Art-1060

35. Reddy JRK, Jyothsna MV, Saleem T S M, Chetty C M S: Review On: Pulsatile Drug Delivery Systems, J. Pharm. Sci. \& Res. Vol.1(4), 2009, 109-115.

36. Wilding IR, Davis SS, Pozzi F, Furlani P, Gazzaniga A. Enteric coated timed release systems for colonic targeting. Int J Pharm 1994; 111: 99-102.

37. Venketesh G. New tools for timed, pulsatile drug delivery. Pharma Formu \& Qual 2005.

38. Jessy S, Amol SB : Oral Multiparticulate Pulsatile Drug Delivery System : A Review, International Research Journal Of Pharmacy, Vol 2 (2) 2011 22-27.

39. Gothoskar AV, Joshi AM, Joshi NH: Pulsatile Drug Delivery Systems: A Review. Drug Del. Tech. 2004; 4: 5-8.
40. Srikanth MV, Sharma GS, Uhumwangho MU, Phani Kumar KS and Ramana Murthy KV: Recent trends in pulsatile drug delivery systems - A review, International Journal of Drug Delivery 2 (2010) 200-212

41. Parmar RD, Parikh RK, Vidyasagar G, Patel DV, Patel CJ and Patel BD: Pulsatile Drug Delivery Systems: An Overview International Journal of Pharmaceutical Sciences and Nanotechnology 2009; 2(3): 605-607.

42. Jain $D$, Raturi $R$, Jain $V$, Bansal $P$ and Singh $R$ : Recent technologies in pulsatile drug delivery systems, Biomatter 1:1, 57-65; July/August/September 2011

43. Ueda Y, Hata T, Yamaguchi H, Kotani M, Ueda S. Development of a novel drug release system, time-controlled explosion system (TES). Part 1: concept and design. J Drug Targeting 1994; 2: 35-44.

44. Singh DK, Poddar AS, Nigade SU, Poddar SS: Pulsatile Drug Delivery System: An Overview, International Journal Of Current Pharmaceutical Review And Research, Volume 2 Issue 2, MayJuly 2011.

45. Wal P, Wal A, Rai AK, Saxena A: Chronopharmaceutics As A Novel Approach For Drug Delivery, Journal Of Pharmaceutical Science And Technology Vol. 1 (2), 2009, 59-62 59

46. Rasve G, Borade G, Deshmukh S And Dr. Tagalpallewar A: Pulsatile Drug Delivery System: Current Scenario, International Journal Of Pharma And Bio Sciences, Vol 2/ Issue 3/ Jul-Sept 2011

47. Kumar PP, Chirag PK: Pulsatile Drug Delivery System: An Overview, Asian Journal Of Pharmaceutical Sciences and Clinical Research, Vol. 1, Issue 1 (2011), 44-51. (tech)

48. Bussemer T, Otto I, Bodmeier R. Pulsatile drug delivery systems Crit Rev. Ther Drug Carrier Syst 2001; 18(5):433-58

49. Lemmer B. Chronopharmacokinetics: implications for drug treatment. J Pharm Pharmacology 1999; 51: 887-890.

50. Veena S Belgamwar, Madhuri V Gaikwad, Ganesh B Patil, Sanjay Surana. Pulsatile drug delivery system. Asian J of Pharmaceutics 2008; 2(3):141-145.

51. Sanders SW, Moore JG. Gastrointestinal chronopharmacology: physiology, pharmacology and therapeutic implications. Pharmacol Ther 1992; 54: .1-15.

52. Amidon GL, Leesman GD. Pulsatile Drug Delivery System. US Patent No. 5,229,131; 1993.

53. Roy, Shahiwala A. Multiparticulate formulation approach to pulsatile drug delivery: Current perspectives. J Control Rele 2009; 134:74-80.

54. Sanvase $S$, Kumar N. Pulsatile drug delivery: Current scenario.CRIPS 2007;8:27-33.

55. Krogel I and Bodmeier R. Floating or pulsatile drug delivery systems based on coated effervescent cores. Int J Pharm. 1999;187:175-184.

56. Punitha S, Sabitha G, Kalal V, Rajasekar S: Floating Drug delivery system- Chronotherapeutic Approach, International research journal of pharmacy, ISSN 2230-8407

57. Sharma $S$ and Pawar A. Low density multiparticulate system for pulsatile release of meloxicam. Int J Pharm. 2006;313:150-158

How to cite this article:

Kaur M and Bala R: Chronotherapy: A Review. Int J Pharm Sci Res. 2013, 4(1); 90-102. 